\title{
ERGODIC PROPERTIES THAT LIFT TO COMPACT GROUP EXTENSIONS
}

\author{
E. ARTHUR ROBINSON, JR.
}

(Communicated by J. Jerry Uhl, Jr.)

\begin{abstract}
Let $T$ and $R$ be measure preserving, $T$ weakly mixing, $R$ ergodic, and let $S$ be conservative ergodic and nonsingular. Let $\tilde{T}$ be a weakly mixing compact abelian group extension of $T$. If $T \times S$ is ergodic then $\tilde{T} \times S$ is ergodic. A corollary is a new proof that if $T$ is mildly mixing then so is $\tilde{T}$. A similar statement holds for other ergodic multiplier properties. Now let $\tilde{T}$ be a weakly mixing type $\alpha$ compact affine $G$ extension of $T$ where $\alpha$ is an automorphism of $G$. If $T$ and $R$ are disjoint and $\alpha$ or $R$ has entropy zero, then $\tilde{T}$ and $R$ are disjoint. $\tilde{T}$ is uniquely ergodic if and only if $T$ is uniquely ergodic and $\alpha$ has entropy zero. If $T$ is mildly mixing and $\tilde{T}$ is weakly mixing then $\tilde{T}$ is mildly mixing. We also provide a new proof that if $\tilde{T}$ is weakly mixing then $\tilde{T}$ has the $K$-property if $T$ does.
\end{abstract}

I. Statement of results. This paper is concerned with a general class of theorems called lifting theorems. A lifting theorem is a theorem of the following sort:

Let $T$ be a weakly mixing measure preserving transformation which satisfies an additional property $P$. If $\tilde{T}$ is a weakly mixing extension of $T$, then $\tilde{T}$ also satisfies $P$.

Throughout this paper an extension will be a compact affine $G$ extension of type $\alpha$, where $G$ is a compact metrizable group and $\alpha$ is a continuous automorphism of $G$ (the definition is given in $§ I I)$. The case where $\alpha$ is trivial is called a compact group extension (abelian extension if $G$ is also abelian).

Lifting theorems are useful for inductively constructing examples of transformations with various properties by lifting these properties to extensions. In the past, lifting theorems have been proven for various kinds of extensions for the following properties $P$ : mixing, Thouvenot (unpublished); $r$-fold mixing, Rudolph [R1]; the $K$-property, Parry [P] (cf. also [T]); and the Bernoulli property, Rudolph [R2]. In [W1], Walters proved a general lifting theorem for abelian extensions concerning the absence of certain invariant sub- $\sigma$-algebras. An easy corollary of this is a lifting theorem for mild mixing in abelian extensions (cf. Corollary 2.1). Berg [B] proved a lifting theorem for group extensions involving quasi-disjointness.

In this paper we prove two new general lifting theorems, and discuss several

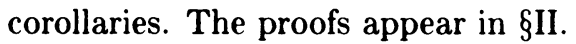

We first consider measure spaces $(X, \mu)$ and $(Y, \nu)$, where $X$ and $Y$ are standard Borel spaces and $\mu$ and $\nu$ are nonatomic Borel probability measures. (We will

Received by the editors January 24, 1986 and, in revised form, September 3, 1986. Some of the results in this paper were presented at the Conference on Smooth Ergodic Theory at the University of Warwick, Coventry, England, July 13, 1986.

1980 Mathematics Subject (lassification (1985 Revision). Primary 28D05, 28D20.

Partially supported by NSF Grant DMS 85-04025. 
usually omit explicit reference to the Borel $\sigma$-algebras.) Let $S$ be a collection of conservative ergodic nonsingular transformations on $(Y, \nu)$. A measure preserving transformation $T$ of $(X, \mu)$ is called an ergodic multiplier for $S$ if the Cartesian product transformation $T \times S$ is ergodic (with respect to $\mu \times \nu$ ) for every $S \in S$. We will denote the set of all ergodic multipliers for $S$ by $\mathcal{M}(S)$.

THEOREM 1. Let $T$ be weakly mixing and an ergodic multiplier for $S$. If $\tilde{T}$ is a weakly mixing compact abelian group extension of $T$ then $\tilde{T}$ is an ergodic multiplier for $S$.

The best known example of an ergodic multiplier property is obtained if $S$ is taken to be the set of all ergodic measure preserving transformations of $(Y, \nu)$. Then $T \in \mathcal{M}(S)$ if and only if $T$ is weakly mixing (cf. [F1]). Furstenberg and Weiss $[\mathbf{F W}]$ studied the case where $S$ is enlarged to include all the transformations $S$ preserving an infinite measure equivalent to $\nu$. In this case they called the transformations $T \in \mathcal{M}(S)$ mildly mixing, and showed that $T$ is mildly mixing if and only if $T$ has no rigid factors. Mild mixing lies properly between weak mixing and mixing. An immediate corollary of Theorem 1 is a new proof of the lifting theorem for mild mixing.

COROLLARY 1.1. If $T$ is mildly mixing and $\tilde{T}$ is a weakly mixing compact abelian group extension of $T$ then $\tilde{T}$ is mildly mixing.

As we noted above, this is also an easy corollary of the work of Walters [W1, Theorem 10] (which actually predates the definition of mild mixing).

If $S$ is enlarged further to include all the ergodic nonsingular transformations of $(Y, \nu)$, it turns out that $\mathcal{M}(S)$ still consists of the mildly mixing transformations (cf. $[\mathbf{A}])$. If, on the other hand, a smaller set is taken to be $S$, then $\mathcal{M}(S)$ may have a more interesting characterization. Such cases were studied by Aaronson [A], who showed that the transformations $T \in \mathcal{M}(S)$ satisfy a certain mixing condition (between weak and mild mixing). The property of belonging to $\mathcal{M}(S)$ is a spectral property; if $\sigma_{T}$ denotes a measure of maximal spectral type for $T$, and if for $S \in$ $S, \Lambda_{S}$ denotes the set of eigenvalues for $S$, then $T \times S$ is ergodic if and only if $\sigma_{T}\left(\Lambda_{S}\right)=0$. Theorem 1 says that all such ergodic muliplier properties satisfy lifting theorems.

The second general lifting theorem which we prove in this paper involves Furstenberg's notion of disjointness [F1]. Let $T$ and $R$ be ergodic measure preserving transformations of $(X, \mu)$ and $(Z, \eta)$ respectively, where again we consider $(X, \mu)$ and $(Z, \eta)$ as standard Borel spaces with $\mu$ and $\eta$ nonatomic Borel probability measures. Let $\varsigma$ be a $T \times R$ invariant Borel probability measure on $X \times Z$ (with the product Borel structure), such that for Borel sets $E \subset X$ and $F \subset Z, \varsigma(E \times Z)=\mu(E)$ and $\zeta(X \times F)=\eta(F)$. The measure $\varsigma$ is called a joining of $T$ and $R$. The transformations $T$ and $R$ are called disjoint if the only joining $\zeta$ of $T$ and $R$ is the product measure $\mu \times \eta$. For a collection $R$ of ergodic measure preserving transformations we denote by $R^{\perp}$ the set of all $T$ disjoint from every $R \in R$. We obtain the following lifting theorem for disjointness.

THEOREM 2. Let $T$ be weakly mixing and belong to $R^{\perp}$. If $\tilde{T}$ is a weakly mixing type $\alpha$ compact affine extension of $T$, where $\alpha$ is a group automorphism with entropy zero, then $\tilde{T}$ also belongs to $R^{\perp}$. 
In the special case of a group extension, this theorem follows from a similar theorem due to Berg $[\mathbf{B}]$, concerning the more general notion of quasi-disjointness. Our proof of Theorem 2 is based on Proposition 3 below which generalizes some well-known results about invariant measures for extensions.

We now list some corollaries of Theorem 2. From [Pi] it follows that $T$ has the $K$ property if and only if it is disjoint from every transformation $R$ with entropy zero. Thus, Theorem 2 gives a new proof of the fact (cf. [P and $\mathbf{T}]$ ) that the $K$-property satisfies a lifting theorem. This proof is particularly simple in the group extension case. Also, since $T$ is mildly mixing if and only if it is disjoint from every rigid transformation $R$ [W1, Corollary 9.1], we obtain (Corollary 2.1) a lifting theorem for mild mixing in compact affine extensions.

II. Proofs. Let $T$ be a nonsingular transformation of a $(X, \mu)$. Let $G$ be a compact separable group, with its natural Borel structure, and Haar measure $\gamma$. Let $\varphi: X \rightarrow G$ be a Borel function and let $\alpha$ be a continuous automorphism of $G$. We define a transformation $\tilde{T}$ of $(\tilde{X}, \tilde{\mu})=(X \times G, \mu \times \gamma)$ by

$$
\tilde{T}(x, g)=(T x, \varphi(x) \alpha(g)) .
$$

We call the transformation $\tilde{T}$ a compact affine $G$ extension of $T$ of type $\alpha$. (Warning: $\tilde{T}$ is given many different names in the literature.) $\tilde{T}$ is always nonsingular, and it is measure preserving whenever $T$ is a measure preserving.

If $\alpha$ has entropy zero with respect to Haar measure (denoted $h(\alpha)=0$ ), we say the extension is relatively zero entropy. If $\alpha$ is the identity automorphism of $G$ then we call $\tilde{T}$ a compact group extension (or $G$ extension). In this case, if $G$ is abelian then $\tilde{T}$ is called an abelian extension of $T$. The function $\varphi$ in (1) is called a cocycle over $T$.

A number $\lambda$ is called an eigenvalue for $T$ if there is a bounded complex Borel function $f$ such that $f(T x)=\lambda f(x)$. The function $f$ is called an eigenfunction corresponding to $\lambda$. The transformation $T$ is ergodic if the only eigenfunctions corresponding to the eigenvalue 1 are the constant functions. For conservative ergodic $T$, each eigenvalue $\lambda$ satisfies $|\lambda|=1$, the corresponding eigenfunction is unique up to constant multiples, and $|f|$ is constant $\mu$-almost everywhere.

If $T$ is ergodic, and if in addition it has no nonconstant eigenfunctions, then $T$ is said to have continuous spectrum. For measure preserving transformations $T$, continuous spectrum is equivalent to weak mixing (cf. $[\mathbf{H}]$ ).

For Theorem 1 we assume that $G$ is compact abelian and $\alpha$ is trivial, so $\tilde{T}$ is an abelian extension. Let $\hat{G}$ denote the dual group of $G$, i.e. the set of continuous homomorphisms $\chi: G \rightarrow S^{1}$, where $S^{1}=\{z \in \mathbf{C}:|z|=1\}$. For $\chi \in \hat{G}$, the cocycle $\varphi$ is called $\chi$-trivial if there exists a measurable function $\psi: X \rightarrow S^{1}$ such that

$$
(\chi \circ \varphi)(x)=\psi(T x) \psi^{-1}(x) \text {. }
$$

It is called almost- $\chi$-trivial if there exists $\lambda \in S^{1}$ and $\psi: X \rightarrow S^{1}$ such that

$$
\lambda^{-1}(\chi \circ \varphi)(x)=\psi(T x) \psi^{-1}(x) \text {. }
$$

LEMMA 1. Suppose $T$ is nonsingular and ergodic on $(X, \mu)$ and that $G$ is abelian. If $\tilde{T}$ is a $G$ extension of $T$ with cocycle $\varphi$, then $\tilde{T}$ is ergodic if and only if $\varphi$ is not $\chi$-trivial for any $\chi \in \hat{G}, \chi \neq 1$. In addition, if $T$ has continuous spectrum, 
then $\tilde{T}$ has continuous spectrum if and only if $\varphi$ is not almost- $\chi$-trivial for any $\chi \neq 1$.

This lemma is well known so we omit the proof. The following is the main lemma for Theorem 1. It is also somewhat interesting in its own right.

LEMMA 2. Let $T$ and $S$ be nonsingular transformations on $(X, \mu)$ and $(Y, \nu)$ such that $T \times S$ is ergodic. Let $\varphi: X \rightarrow G$ be a cocycle over $T$ which is not almost$\chi$-trivial for any $\chi$ in $\hat{G}$. Define $\varphi^{\prime}: X \times Y \rightarrow G$ by $\varphi^{\prime}(x, y)=\varphi(x)$. Then $\varphi^{\prime}$, as a cocycle over $T \times S$, is not $\chi$-trivial for any $\chi \in \hat{G}$.

Proof. Suppose that $\varphi^{\prime}$ is $\chi$-trivial. Then

$$
(\chi \circ \varphi)(x)=\left(\chi \circ \varphi^{\prime}\right)(x, y)=\psi(T x, S y) \psi^{-1}(x, y)
$$

and

$$
(\chi \circ \varphi)(x)=\left(\chi \circ \varphi^{\prime}\right)(x, S y)=\psi\left(T x, S^{2} y\right) \psi^{-1}(x, S y) .
$$

Thus if we define

$$
\omega(x, y)=\psi(x, S y) \psi^{-1}(x, y)
$$

we have that $\omega((T \times S)(x, y))=\omega(x, y)$, and so by the ergodicity of $T \times S, \omega(x, y)=$ $\omega_{0}$. By (5), this implies that

$$
\psi(x, S y)=\omega_{0} \psi(x, y)
$$

Now, for $\mu$-almost every $x,(6)$ implies that $\psi(x, \cdot)$ is an eigenfunction for $S$ corresponding to the eigenvalue $\omega_{0}$. Since $S$ is ergodic, this eigenfunction is unique up to constant multiples. This allows us to write $\psi(x, y)=\theta(x) \xi(y)$, where $\xi(S y)=\omega_{0} \xi(y)$. From (4) and (5) we have

$$
\begin{aligned}
(\chi \circ \varphi)(x) & =\theta(T x) \xi(S y) \theta^{-1}(x) \xi^{-1}(y) \\
& =\omega_{0} \theta(T x) \theta^{-1}(x) .
\end{aligned}
$$

But this clearly implies that $\varphi$ is almost- $\chi$-trivial.

PROOF OF THEOREM 1 . Since $\tilde{T}$ is weakly mixing, the corresponding cocycle $\varphi$ is not almost- $\chi$-trivial for any $\chi$. By assumption, $T \times S$ is ergodic, and clearly $\tilde{T} \times S$ is a group extension of $T \times S$ corresponding to the cocycle $\varphi^{\prime}(x, y)=\varphi(x)$. By Lemma $2, \varphi^{\prime}$ is not $\chi$-trivial for any $\chi \in \hat{G}$, and so by Lemma $1, \tilde{T} \times S$ is ergodic.

Notice that the proof of Theorem 1 depends on the fact that $G$ is abelian. Our work on Theorem 2 began as an attempt to extend Corollary 1.1 to the case where $G$ is nonabelian. The proof, based on Proposition 3 below, turns out to be even more general.

Before discussing the proposition we have the following definition. For a Borel measure $\zeta$ on a product of standard Borel spaces $X \times Z$, we define the marginals $\varsigma_{X}$ and $\varsigma_{Z}$ as the measures on $X$ and $Z$ respectively satisfying $\varsigma_{X}(E)=\varsigma(E \times Z)$ and $\varsigma Z(F)=\varsigma(X \times F)$.

Proposition 3. Suppose $T$ is ergodic on $(X, \mu)$. Let $\tilde{T}$ be an ergodic relatively zero entropy compact affine extension of $T$ on $(\tilde{X}, \tilde{\mu})$. If $\varsigma$ is a $\tilde{T}$ invariant Borel probability measure on $\tilde{X}$ with $\varsigma_{X}=\mu$, then $\varsigma=\mu \times \gamma=\tilde{u}$. 
Many cases of this proposition seem to be well known. The group extension case is essentially due to Furstenberg (cf. [F1, proof of Theorem I.4], also [F2, Proposition 3.10]). In [JR] an easy argument is given for group extensions, providing very simple proofs of Theorem 2 and its corollaries in that case.

When $\alpha \neq$ Id the situation becomes more complicated. In [W2], Walters proves Lemma 3 for three special types of relatively zero entropy compact affine extensions. These are

(i) $G$ is compact abelian and $\alpha^{n}$ is unipotent for some $n$,

(ii) $G$ is finite, and

(iii) $G$ is a compact simple Lie group.

The proof uses deep structure theorems for compact group automorphisms (cf. [W2, T]). We will show how to derive the general case from (i), (ii), and (iii) using further arguments from [W2] and the following lemma.

Let $T$ be a measure preserving transformation $T$ of $(X, \mu)$. Let $\mathcal{F}=\left\{F_{x}: x \in X\right\}$ be a measurable family of measure preserving transformations of $(Z, \eta)$ (where we allow $Z$ to be finite). Let $(\tilde{X}, \tilde{\mu})=(X \times W, \mu \times \eta)$ and define

$$
\tilde{T}(x, z)=\left(T x, F_{x} z\right) .
$$

The transformation $\tilde{T}$ in (6) is called a skew product of $T$ and $\mathcal{F}$ or a skew product over $T$. Note that a compact affine extension is a special case of a skew product. We say that the skew product $\tilde{T}$ over $T$ has the measure lifting property if for any $\tilde{T}$ invariant measure $\varsigma$ with $\varsigma_{X}=\mu$ we have $\varsigma=\mu \times \eta$. Proposition 3 says this property holds for relatively zero entropy compact affine extensions.

Let $\tilde{T}_{0}=T$ and for $n=0,1,2 \ldots$, let $\tilde{T}_{n+1}$ be a skew product of $\tilde{T}_{n}$ and $\mathcal{F}_{n}$, where $\mathcal{F}_{n}$ acts on $\left(Z_{n}, \eta_{n}\right)$. Let $Z$ be the product of the spaces $Z_{n}$ and let $\eta$ be the product measure. We define the inverse limit $\tilde{T}$ of the sequence $\tilde{T}_{n}$ on $(\tilde{X}, \tilde{\mu})=(X \times Z, \mu \times \eta)$ by

$$
\tilde{T}\left(x, z_{1}, z_{2}, \ldots\right)=\left(T x, F_{x}^{1} z_{1}, F_{x, z_{1}}^{2} z_{2}, \ldots, F_{x, z_{1}, \ldots, z_{j-1}}^{j} z_{j}, \ldots\right),
$$

where $\mathcal{F}_{n}=\left\{F_{x^{\prime}}^{n}: x^{\prime}=\left(x, z_{1}, \ldots, z_{n-1}\right)\right\}$. Note that $\tilde{T}$ is itself a skew product over $T$.

LEMMA 3. Let $\tilde{T}$ be an inverse limit of $\tilde{T}_{n}$. Suppose that $\tilde{T}_{n+1}$ has the measure lifting property with respect to $\tilde{T}_{n}$ for each $n$. Then $\tilde{T}$ has the measure lifting property with respect to $T$.

Proof. Let $\left(\tilde{X}_{n}, \tilde{\mu}_{n}\right)=\left(X \times W_{1} \times \cdots \times W_{n}, \mu \times \eta_{1} \times \cdots \times \eta_{n}\right)$. By definition, a measure $\varsigma$ on $\tilde{X}$ is a product measure if it induces product measure on each finite cylinder set. Thus the lemma will follow if we show that for each $n, \varsigma_{\tilde{X}_{n}}=\tilde{\mu}_{n}$, since $\tilde{\mu}_{n}$ is a product measure.

We have $\varsigma_{\tilde{X}_{0}}=\varsigma_{X}=\mu=\tilde{\mu}_{0}$ and clearly $\varsigma_{\tilde{X}_{n}}$ is $\tilde{T}_{n}$ invariant. By induction, we suppose that $\varsigma_{\tilde{X}_{n-1}}=\tilde{\mu}_{n-1}$. Then $\left(\varsigma_{\tilde{X}_{n}}\right) \tilde{X}_{n-1}=\tilde{\mu}_{n-1}$ so that $\varsigma_{\tilde{X}_{n}}$ satisfies the assumptions on $\varsigma_{n}$. Then our hypotheses imply $\varsigma_{\tilde{X}_{n}}=\tilde{\mu}_{n}$ which completes the induction step.

ProOF OF PROPOSITION 3. Walters shows in [W2] (cf. also [L2]) that in general, a type $\alpha$ extension $\tilde{T}$ of $T$ can be factored into a series of three extensions (i.e. is an extension of an extension of an extension). Each of these three extensions 
is an inverse limit of a (possibly finite) sequence of extensions $\tilde{T}_{n}$, where $\tilde{T}_{n}$ is a $G_{n}$ extension of type $\alpha_{n}$ of $\tilde{T}_{n-1}$. Each of these type $\alpha_{n}$ extensions is either of the form (i), (ii) or (iii), or $\alpha_{n}$ is an ergodic automorphism of $G_{n}$.

If $\alpha_{n}$ is an ergodic group automorphism then it has the $K$-property (cf. [T]) and so $h\left(\alpha_{n}\right)>0$. Thus if $h(\alpha)=0$ (the relatively zero entropy case), all the ergodic steps in each inverse limit must be trivial. The proposition now follows from cases (i), (ii), and (iii) and Lemma 3.

Proposition 3 is never true in the case $h(\alpha)>0$. Indeed it follows from Lind [L1] that if $\alpha$ is ergodic, every type $\alpha$ extension $\tilde{T}$ of $T$ is conjugate to a direct product transformation $T \times \alpha$. The conjugating map on $\tilde{X}$ has the form $P(x, g)=\left(x, Q_{x}(g)\right)$, where $Q_{x}$ preserves $\gamma$. Since ergodic group automorphisms are never uniquely ergodic (consider the point mass at $e \in G$ ), there exists an $\alpha$ invariant measure $\lambda \neq \gamma$, and the pull back of $\mu \times \lambda$ via $P$ is the desired counterexample.

We note the following corollary to Proposition 3 which is similar to results of Walters [W2] and Furstenberg [F2].

COROLLARY 3.1. Let $T$ be a uniquely ergodic homeomorphism of a compact metric space $X$. An ergodic compact affine extension $\tilde{T}$ of $T$ is uniquely ergodic if and only if it is relatively zero entropy.

We are now ready for the following.

ProOF OF THEOREM 2. Let $\varsigma$ be a joining of $\tilde{T}$ and $R$. Then $\zeta$ is a $\tilde{T} \times R$ invariant measure with $\zeta_{Z}=\eta$ and $\varsigma_{\tilde{X}}=\mu \times \gamma$. Also, since $\tilde{T}$ is weakly mixing, $\tilde{T} \times R$ is a $\mu \times \gamma \times \eta$ ergodic extension of $T \times R$. Now $\varsigma_{X}=\left(\varsigma_{X}\right)_{X}=(\mu \times \gamma)_{X}=\mu$ and $\zeta_{Z}=\eta$, so $\left(\zeta_{X \times Z}\right)_{X}=\varsigma_{X}=\mu$ and $\left(\zeta_{X \times Z}\right)_{Z}=\zeta_{Z}=\eta$. Thus $\zeta_{X \times Z}$ is a joining of $T$ and $R$. Since $T$ and $R$ are assumed to be disjoint, $\zeta X \times Z=\mu \times \eta$. It follows from Lemma 3 that $\zeta=\mu \times \gamma \times \eta$.

Already, this is enough to prove Corollary 2.1 in the relatively zero entropy case. However, we can extend Theorem 2 to some cases where $h(\alpha)>0$. Namely, suppose $R$ consists entirely of transformations with entropy zero and $\alpha$ is ergodic. By the work of Lind [L1] mentioned above, $\tilde{T}$ is isomorphic to $T \times \alpha$. Since $\alpha$ has the $K$-property (because it is ergodic), it is easy to see that $\tilde{T} \in R^{\perp}$. We also note that if $R$ is rigid then it has entropy zero. By combining these observations with the proofs of Theorem 2 and Lemma 4 we obtain the following.

COROLLARY 2.1. If $T$ is mildly mixing and $\tilde{T}$ is a weakly mixing compact affine extension of $T$, then $\tilde{T}$ is mildly mixing.

COROLLARY 2.2. If $T$ and $R$ are disjoint and $\tilde{T}$ and $\tilde{R}$ are weakly mixing relatively zero entropy compact affine extensions of $T$ and $R$, then $\tilde{T}$ and $\tilde{R}$ are disjoint.

For the sake of completeness we include the following.

COROllaRY $2.3[\mathbf{P}, \mathbf{T}]$. If $T$ has the $K$-property and $\tilde{T}$ is a weakly mixing compact affine extension of $T$, then $\tilde{T}$ has the $K$-property.

ACKNOWLedgment. The author wishes to thank J. Aaronson for suggesting the line of inquiry leading to Theorem 1 and P. Walters for pointing out [W2]. 
ADDED IN PROOF. In the group extension case, J. Coquet and P. Liardet ( $A$ metric study involving independent sequences, J. Analyse Math. (to appear)), have independently given the same proof of Theorem 2 and Corollary 2.3 .

\section{REFERENCES}

[A] J. Aaronson, Category theorems for ergodic multiplier properties, Israel J. Math. 51 (1985), $151-162$.

[B] K. Berg, Quasi-disjointness in ergodic theory, Trans. Amer. Math. Soc. 162 (1971), 71-87.

[F1] H. Furstenberg, Disjointness in ergodic theory, minimal sets, and a problem in diophantine approximation, Math. Systems Theory 1 (1967), 1-49.

[F2] _ - Recurrence in ergodic theory and combinatorial number theory, Princeton Univ. Press, Princeton, N. J., 1981.

[FW] H. Furstenberg and B. Weiss, The finite multipliers of infinite ergodic transformations, Lecture Notes in Math., vol. 668, Springer-Verlag, Berlin and New York, 1978, pp. 127-132.

[H] P. Halmos, Lectures on ergodic theory, Publ. Math. Soc. Japan, Tokyo, 1956.

[JR] A. del Junco and D. Rudolph, On ergodic actions whose joinings are graphs, Report PM-R8408, Center for Mathematics and Computer Science, Amsterdam, 1984.

[L1] D. Lind, The structure of skew products with ergodic group automorphisms, Israel J. Math. 28 (1977), 205-248.

[L2] _ _ Ergodic affine transformations are loosely Bernoulli, Israel J. Math. 30 (1978), 335-338.

[P] W. Parry, Ergodic properties of transformations and flows on nilmanifolds, Amer. J. Math. 91 (1969), 757-771.

[Pi] M. S. Pinsker, Dynamical systems with completely positive or zero entropy, Dokl. Akad. Nauk. SSSR 133 (1960), 1025-1026; English transl., Soviet Math. Dokl. 1 (1961), 937-938.

[R1] D. Rudolph, K-fold mixing lifts to weakly mixing isometric extensions, Ergodic Theory and Dynamical Systems 5 (1985), 445-447.

[R2] _ Classifying the isometric extensions of a Bernoulli shift, J. Analyse Math. 34 (1978), 36-60.

[T] R. K. Thomas, Metric properties of transformations of $G$-spaces, Trans. Amer. Math. Soc. 160 (1971), 103-117.

[W1] P. Walters, Some invariant $\sigma$-algebras for measure preserving transformations, Trans. Amer. Math. Soc. 163 (1972), 357-367.

[W2] _ Some transformations having a unique measure with maximal entropy, Proc. London Math. Soc. 3 (1974), 500-516.

Department of Mathematics, University of Pennsylvania, Philadelphia, PENNSYLVANIA 19104

Current address: Department of Mathematics, George Washington University, Washington, D.C. 20052 